\title{
Accelerator technology and SPECT developments for BNCT
}

\author{
Alejandro A. Valda ${ }^{* 1,2}$, Javier Bergueiro, ${ }^{1,3}$, Matías Baldo ${ }^{1}$, Daniel Cartelli ${ }^{1,2,3}$, Walter \\ Castell', Javier Gómez Asoia ${ }^{1}$, Javier Padulo', Juan Carlos Suarez Sandín', \\ Marcelo Igarzábal ${ }^{1}$, Julián Erhardt ${ }^{1}$, Daniel O. Mercuri ${ }^{1}$, Daniel M. Minsky ${ }^{1,2,3}$, José \\ María Kesque $^{1}$, María Eugenia Capoulat ${ }^{1,2,3}$, María S. Herrera ${ }^{1,2,3}$, Sara González ${ }^{1,3}$, \\ Héctor Somacal $^{1,2}$, Mario E. Debray ${ }^{1,2}$, Mariela F. del Grosso ${ }^{1,3}$, Leonardo \\ Gagetti $^{1,2,3}$, Manuel Suarez Anzorena1, Oscar García Carranza1, Nicolás Cánepa1, \\ Santiago Girola ${ }^{1,2}$, Marcelo Gun ${ }^{4}$, Lucila Rogulich ${ }^{1,2}$, Andrés J. Kreiner ${ }^{1,2,3}$ \\ 1 Gerencia de Investigación y Aplicaciones, Comisión Nacional de Energía Atómica, Argentina \\ 2 Escuela de Ciencia y Tecnología, Universidad Nacional de San Martín, San Martín, Argentina \\ 3 Consejo Nacional de Investigaciones Científicas y Técnicas, Buenos Aires, Argentina \\ 4 Facultad de Ingeniería, Universidad de Buenos Aires Av. Paseo Colón 850, Buenos Aires, Argentina \\ E-mail: avalda@unsam.edu.ar
}

\begin{abstract}
Accelerator-Based Boron Neutron Capture Therapy (AB-BNCT) is establishing itself worldwide as the future modality to start the phase of in-hospital facilities. There are projects in Russia, UK, Italy, Japan, Israel and Argentina to develop AB-BNCT around different types of accelerators. In particular, the present status and recent progress of the Argentine project for the development of a Tandem-Electrostatic-Quadrupole (TESQ) accelerator is presented. Different working areas are treated: high-power ion sources, acceleration tubes, transport of intense beams, beam diagnostics, high-power targets, ${ }^{9} \mathrm{Be}(\mathrm{d}, \mathrm{n})$ reaction as a possible neutron source and treatment room design. A complete test stand was built and commissioned for intense proton beam production and characterization. Beams of 10 to $30 \mathrm{~mA}$ have been produced and transported during variable periods of operation by means of a pre accelerator and an electrostatic quadrupole doublet to a suppressed Faraday cup. The beam diagnostics has been performed through the observation with digital cameras of induced fluorescence in the residual gas. A 200 $\mathrm{kV}$ TESQ accelerator prototype has been constructed and is under test and a $600 \mathrm{keV}$ prototype is under construction. Self consistent space charge beam transport simulations have been performed and compared with experiments. In addition to the traditional ${ }^{7} \mathrm{Li}(\mathrm{p}, \mathrm{n})$ reaction, ${ }^{9} \mathrm{Be}(\mathrm{d}, \mathrm{n})$ using a thin Be target has been thoroughly studied as a candidate for a possible neutron source for deep seated tumors, showing a satisfactory performance. A treatment room complying with regulations has been designed. Finally we present advances in the development of a SinglePhoton Emission Computed Tomography (SPECT) system for online dosimetry during a BNCT treatment.
\end{abstract}

X Latin American Symposium on Nuclear Physics and Applications (X LASNPA)

December 1-6 2013

Montevideo, Uruguay

\footnotetext{
* Speaker 


\section{Introduction}

Boron Neutron Capture Therapy (BNCT) [1,2] appears as a therapeutic alternative in cases where tumors are spatially not well defined -i.e., have diffuse limits and regions with partial infiltration- or when they do not respond to conventional chemo- or radiotherapy or when the tumor location or its degree of dissemination make a meaningful surgical approach difficult, if not impossible. BNCT is performed in two steps and therefore it is characterized as a binary approach. The first step consists in giving the patient a tumor-selective drug that acts as a carrier for ${ }^{10} \mathrm{~B}$. Once the tumor cells are loaded with ${ }^{10} \mathrm{~B}$, the patient is irradiated with an epithermal neutron beam. Epithermal energy is necessary for reaching deep seated tumors with thermal energies at which the capture cross section (in this case, of ${ }^{10} \mathrm{~B}$ ) is very large. The capture reaction produces two high-LET (linear energy transfer) ions that release more than $2 \mathrm{MeV}$ of energy within a combined range of the order of $10 \mu \mathrm{m}$, a typical cell size. This binary procedure of BNCT is a quite unique feature and achieves a cell level targeting provided that good selectivity of the boron-carrier drug is obtained. Current drugs used in clinical BNCT attain a concentration ratio between tumor and healthy tissue of approximately 3 .

At present, almost all BNCT treatment centers worldwide are associated to nuclear reactors. However it is recognized that the advancement of BNCT requires neutron sources suitable for installation in hospital environments. In this scenario, accelerator-based neutron sources, installed in specialized cancer centers, will play a decisive role in the future of BNCT. Therefore several programs, whose number has increased during the last few years, are dedicated to the development of accelerator-based BNCT (AB-BNCT): two in Japan [3,4], two in Russia [5,6], one in Israel [7], one in Italy [8], one in the United Kingdom [9] and one in Argentina [10].

There are several nuclear reactions of interest for AB-BNCT, the two most exploited ones in current $\mathrm{AB}-\mathrm{BNCT}$ programs are ${ }^{7} \mathrm{Li}(\mathrm{p}, \mathrm{n}){ }^{7} \mathrm{Be}$ and ${ }^{9} \mathrm{Be}(\mathrm{p}, \mathrm{n}){ }^{9} \mathrm{~B}$. The lithium reaction was traditionally considered the best due to its neutron yield and because it can produce low energy neutrons at bombarding energies near its threshold $(1.88 \mathrm{MeV})$. However the most awkward properties of lithium for constructing high-power targets are its chemical reactivity, low melting point $\left(180^{\circ}\right)$, low thermal conductivity $\left(85 \mathrm{~W} \cdot \mathrm{m}^{-1} \cdot \mathrm{K}^{-1}\right)$ and management of the radioactive product generated in the reaction $\left({ }^{7} \mathrm{Be}, T_{1 / 2}=53.2\right.$ days), though these are not insurmountable problems $[5,6,7,9]$.

In this paper we report on the present status of an ongoing project to develop a folded Tandem-Electrostatic-Quadrupole (TESQ) accelerator facility for AB-BNCT at the Atomic Energy Commission of Argentina (CNEA) [11]. The progress achieved in the different working areas of the project is presented including an approach for the online determination of the delivered dose to the patient.

\section{The ongoing project on AB-BNCT in Argentina}

The planned facility is based on a vertical folded tandem with electrostatic quadrupoles (TESQ) for acceleration and beam transport. The machine is designed for the ${ }^{7} \mathrm{Li}(\mathrm{p}, \mathrm{n})^{7} \mathrm{Be}$ reaction for operation slightly beyond its resonance at $2.3 \mathrm{MeV}$ (although we are also studying the near threshold condition [21]). It is intended to work in air, to avoid the need for a pressure 
vessel and for an insulating gas installation. The project aims at developing a machine capable of delivering a proton beam of about $2.4 \mathrm{MeV}$ and $30 \mathrm{~mA}$ to irradiate a Li metal (or a refractory $\mathrm{Li}$ compound) target in order to produce the therapeutic neutron beam after appropriate beam shaping. These are the specifications needed to produce sufficiently intense and clean epithermal neutron beams to perform AB-BNCT for deep-seated tumors in less than an hour. Details and discussions on this installation can be found in previous publications [10-13].

The project involves several working areas, namely development of high power ion sources, development of power and voltage generation systems, development of acceleration tubes, transport study of intense beams, implementation of beam diagnostics, implementation of control systems, develpment of high power targets, study and construction of beam shaping assemblies, treatment room design, treatment planning assessment of clinical cases, the ${ }^{9} \mathrm{Be}(\mathrm{d}, \mathrm{n})$ reaction as a possible neutron source and online patient dosimetry.

Since the accelerator has a modular concept it can be progressively scaled up in energy. The different prototypes being under study and in different stages of constructions are a $200 \mathrm{kV}$ single-ended, a $700 \mathrm{kV}$ tandem and a $1.4 \mathrm{MV}$ tandem. They share a number of common features, like the beam acceleration and transport principle (based on a chain of electrostatic quadrupoles), accelerator tubes, power generation systems (for the $700 \mathrm{kV}$ and $1.4 \mathrm{MV}$ prototypes) and high-voltage sources. In particular, extensive numerical calculations for the transport of high intensity beams with space charge effects have been performed for the design of the ion optics [14].
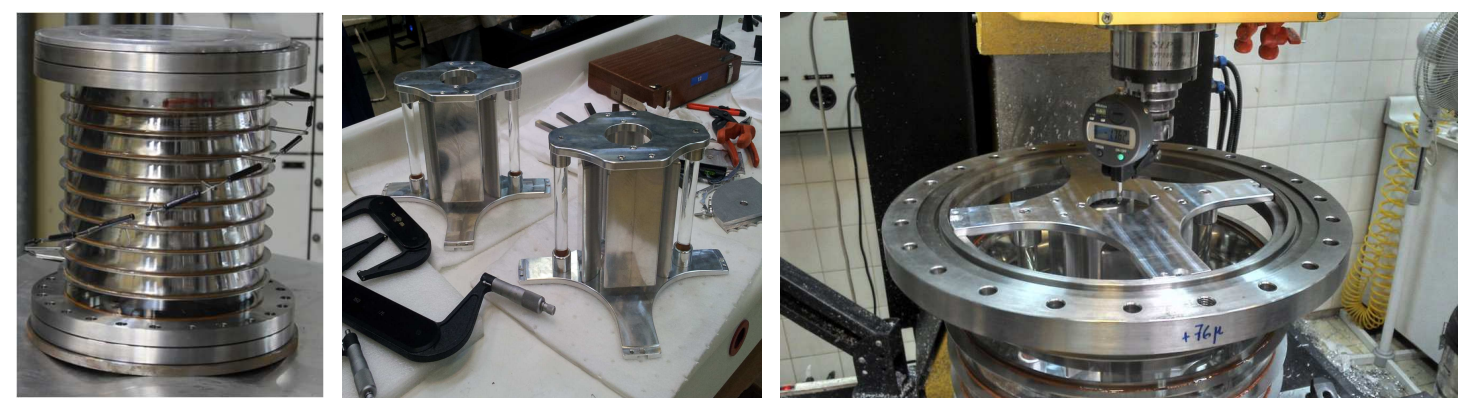

Figure 1: Left: tube section. Center: quadrupole modules. Right: centering the quadrupoles inside the tube.

\subsection{Accelerator tubes and quadrupoles}

Numerical simulations for stress and deformation studies have been performed for a tube design based on glass cylinder slabs with electrodes interleaved and bonded with an appropriate adhesive. A built tube successfully passed the mechanical test, the high voltage test (106 kV applied between ends) and vacuum test $\left(3 \times 10^{-7} \mathrm{mbar}\right)$ [15]. Figure 1 shows a tube section, like those used in the ion source test stand and the $200 \mathrm{kV}$ accelerator, along with its quadrupole modules. Final relevant dimensions in the assembled quadrupoles are within $0.03 \mathrm{~mm}$ precision.

\subsection{The ion source}

A dual-chamber plasma volume source has been constructed and assembled [16]. This source, working with hydrogen, in combination with a pre-acceleration system of electrodes in a test stand has produced an intense proton beam of about $30 \mathrm{~mA}$. The beam intensity is high enough to produce intense fluorescence in the residual gas inside the tube. Digital images were 
acquired, processed and analyzed for extracting relevant beam parameters (figure 2). An iterative calculation gives an initial beam radius of $3.46 \mathrm{~mm}$ and an emittance of $1.20 \mathrm{~mm} \cdot \mathrm{mrad}$.

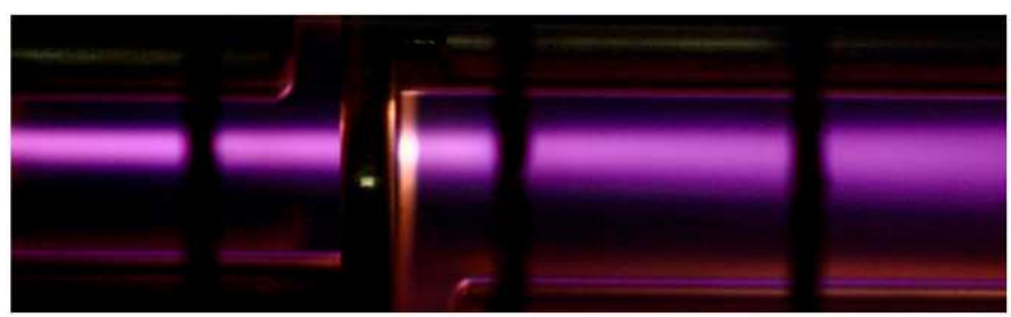

Figure 2: Induced fluorescence in the residual gas produced by a proton beam of $9.5 \mathrm{~mA}$.

\subsection{The $200 \mathrm{kV}$ prototype}

Figure 3.a shows a general view of the $200 \mathrm{kV}$ accelerator with all its peripheral systems high voltage, cooling, vacuum and control- mounted. Part b. shows a detail of the quadrupole chain in place inside the tube. The accelerator has produced stable beams of the order of the $\mathrm{mA}$, figure 3.c shows the first beam successfully produced with an intensity of $3 \mathrm{~mA}$.

a.

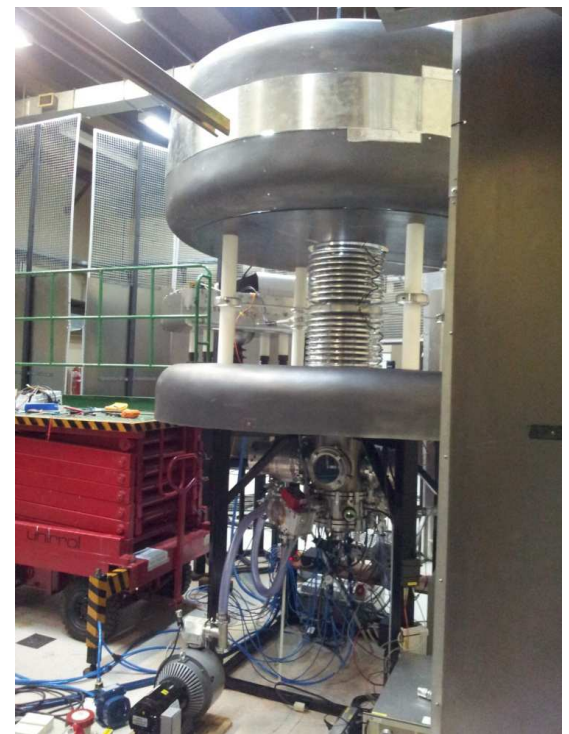

b.

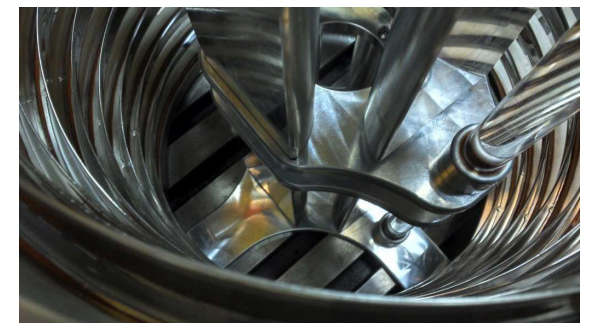

c.

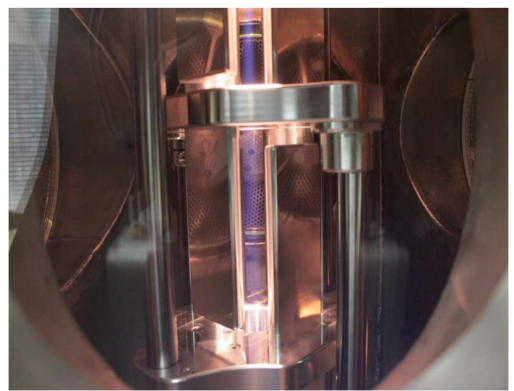

Figure 3: (a.) General view of the $200 \mathrm{kV}$ accelerator. (b.) Quadrupole chain inside the tube. (c.) First beam propagating through the accelerator ( $3 \mathrm{~mA})$.

\subsection{Neutron production target}

As stated above, in a first stage of the accelerator development, the machine is intended to deliver a high intensity beam of deuterons of about $1.4 \mathrm{MeV}$. The aim is that this accelerator works in conjunction with a thin $\mathrm{Be}$ target. In this context the ${ }^{9} \mathrm{Be}(\mathrm{d}, \mathrm{n})^{10} \mathrm{~B}$ reaction was studied as a promising alternative to the ${ }^{7} \mathrm{Li}(\mathrm{p}, \mathrm{n})^{7} \mathrm{Be}$ reaction as an epithermal neutron source for treating deep seated tumors $[17,18]$. In conjunction with an optimized beam shaping assembly, therapeutic doses can be delivered to tumors located up to about $5 \mathrm{~cm}$ in depth with an $8 \mu \mathrm{m}$ target and a deuteron beam of $1.45 \mathrm{MeV}(30 \mathrm{~mA})$ in a single irradiation session of less than 1 hour duration. In addition, the technological advantages of ${ }^{9} \mathrm{Be}(\mathrm{d}, \mathrm{n})$ reaction must be emphasized. They are the low bombarding energies required (about half of the proton energy required for ${ }^{7} \mathrm{Li}(\mathrm{p}, \mathrm{n})$ ), the thermal (melting point: $1287^{\circ}$, thermal conductivity: $190 \mathrm{~W} \cdot \mathrm{m}^{-1} \cdot \mathrm{K}^{-1}$ ) 
and mechanical superiority of a metallic Be target as compared to a Li one and the absence of residual radioactivity.

For the target construction we have achieved stable beryllium deposits on tungsten and molybdenum. However it is necessary to improve the surface preparation in order to get a smoother beryllium surface. A micro channel geometrical was proposed as heath sink for the target and a prototype is under construction $[19,20]$.

\subsection{Patient treatment room design}

A preliminary design of the treatment room, based on Monte Carlo numerical simulations with the MCNP code, has been proposed. It considers an epithermal neutron beam produced via the ${ }^{7} \mathrm{Li}(\mathrm{p}, \mathrm{n})^{7} \mathrm{Be}$ reaction at $2.3 \mathrm{MeV}$ proton energy and $30 \mathrm{~mA}$ and a shielded beam shaping assembly (BSA). The room has an internal footprint of $5 \mathrm{~m} \times 5 \mathrm{~m}$ and is accessed through a maze-like corridor. The wall material is standard concrete $(1.5 \mathrm{~m}$ thickness $)$ with a slab of $\mathrm{Li}_{2} \mathrm{Co}_{3}\left(2 \mathrm{~cm}\right.$ thickness). Layers of water, $\mathrm{Li}_{2} \mathrm{Co}_{3}$, lithiated polyethylene and lead are used for shielding the BSA. The numerical calculations indicate that the proposed shielding for both BSA and treatment room fulfils (in order of magnitude) the dose restrictions required by national regulations $\left(10^{-4} \mathrm{mSv} / \mathrm{h}\right.$ for general public and $3 \times 10^{-3} \mathrm{mSv} / \mathrm{h}$ for occupational workers) considering maximum workload and continuous occupation of adjacent dependencies $[22,23]$.

\section{SPECT-based online dosimetry in BNCT}

\subsection{Statement of the problem}

In BNCT, as in any other radiotherapy approach, a very important issue is the knowledge of the delivered dose to the patient. Dosimetry in BNCT is complicated by the existence of multiple secondary radiations mainly produced by neutron interactions with nuclides other than ${ }^{10} \mathrm{~B}$ present both, in the patient body and in its surrounding environment (e.g. ${ }^{14} \mathrm{~N}(\mathrm{n}, \mathrm{p}){ }^{14} \mathrm{C}$, $\left.{ }^{1} \mathrm{H}(\mathrm{n}, \gamma)^{2} \mathrm{H},{ }^{1} \mathrm{H}(\mathrm{n}, \mathrm{n})\right)$. In the case of BNCT with reactors it is also necessary to consider the gamma radiation originated in the reactor core that emerges from the beam port. Nevertheless the absorbed dose in the tumor is dominated by the ${ }^{10} \mathrm{~B}$ capture reaction which, with currently achieved ${ }^{10} \mathrm{~B}$ concentrations in tumor, accounts for about $80 \%$ of the total dose. Therefore, the boron component dose depends not only on the neutron field but also on the distribution of boron, both in tumor and healthy tissues.

Figure 4 schematically shows the typical steps followed at present for performing patient dosimetry in BNCT. On the one hand, the neutron field is determined and characterized by Monte Carlo calculations and measurements prior to the patient treatment. On the other hand, ${ }^{10} \mathrm{~B}$ concentration in tumor should be determined for each patient. This is obtained from in situ boron concentration measurements on blood samples. Due to the boron carrier drug pharmacokinetics, this is performed on samples taken before, after and, sometimes, during the neutron irradiation. Tumor-to-blood and healthy tissue-to-blood ${ }^{10} \mathrm{~B}$ concentrations are inferred from available statistical data of biodistribution studies of the drug. These studies are conducted on other individuals and also on animal models; the typical value of the ratio $3: 1$ for ${ }^{10} \mathrm{~B}$ concentration in tumor and healthy tissue, is obtained in this way. Finally, the neutron flux and the estimated boron concentration distribution are used to obtain the dose distribution of the patient. 


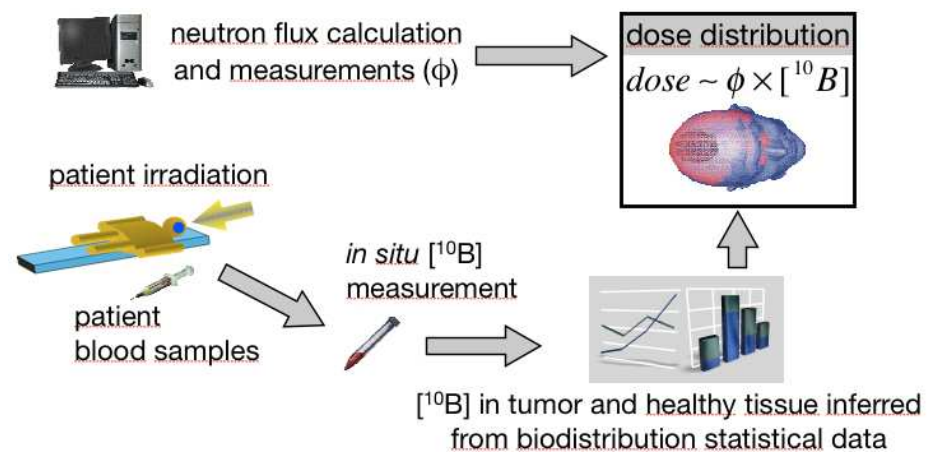

Figure 4: Main typical steps followed at present for performing patient dosimetry in BNCT.

It can be observed that this indirect methodology has large uncertainties due to intrapatient and patient-to-patient variability; it provides thus only very uncertain global dose values and it is not possible, in particular, to consider variations in the dose due to non-homogeneities in ${ }^{10} \mathrm{~B}$ concentrations. Hence a better measurement of ${ }^{10} \mathrm{~B}$ concentration during a BNCT treatment will result in a better knowledge of the response to the treatment.

\subsection{System design and testing of a prototype}

To tackle the dosimetric problem in BNCT, we proposed a methodology that takes advantage of the characteristic gamma radiation emitted during the capture reaction in ${ }^{10} \mathrm{~B}[24$, $25,26]$. This process occurs in $94 \%$ of the captures where the resulting ${ }^{7} \mathrm{Li}$ ion is emitted in an excited state decaying immediately through a $478 \mathrm{keV}$ prompt gamma ray. External detection of these photons is possible since its linear attenuation coefficient in soft tissues is $\sim 0.1 \mathrm{~cm}^{-1}$. Moreover we proposed a tomographic detection system analogue to that used in the nuclear medicine imaging modality known by the acronym SPECT (for single-photon emission computed tomography). This approach is non-invasive, patient-specific and can be performed online, i.e. during the BNCT irradiation. In addition, the tomographic principle gives access to three-dimensional information of the dose distribution. Other approaches have also been adopted for BNCT online dosimetry based on the $478 \mathrm{keV}$ gamma ray detection, some of them were implemented in a BNCT facility [27] and others comprises conceptual studies or basic detector developments $[28,29,30]$.

Despite the widespread application of SPECT in nuclear medicine, the use of such conventional diagnostic system for online BNCT dosimetry is not possible. The energy of the gamma ray of interest and the gamma and neutron background present in a BNCT treatment room demand the development of a dedicated system whose detectors have an energy resolution better than $5 \%$ (to separate the $478 \mathrm{keV}$ and $511 \mathrm{keV}$ peaks), high intrinsic detection efficiency (for collecting enough data during the duration of the patient irradiation) and compact size (for practical reasons related to gamma and neutron shielding). The collimator of the detection system must be designed to cope with the relatively high energy photons and must provide high efficiency (again, for allowing rapid data collection) while providing good spatial resolution for generating useful dose distributions.

Based on Monte Carlo numerical simulations with the MCNP code, we have shown in a previous work [24] the feasibility of a system that produces tomographic slices $(1 \mathrm{~cm}$ spatial resolution) capable to recover the ${ }^{10} \mathrm{~B}$ concentration in a $2 \mathrm{~cm}$ diameter cylindrical tumor model immersed in a $16 \mathrm{~cm}$ cylinder containing an aqueous uniform distribution of boron (tumor-to- 
background concentration: 4:1). Taking into consideration the limited number of counts imposed by the patient treatment time and the system detection efficiency, an uncertainty of around $10 \%$ is expected for the tumor concentration. The tomographic reconstruction was performed by means of a statistical iterative algorithm called Expectation Maximization Maximum Likelihood (EM-ML) [31]. The system matrix needed for the reconstruction was calculated by Monte Carlo simulations using MCNP, taking into account attenuation and scatter in collimator, photoelectric absorption in the scintillator and attenuation in the phantom.

A prototype of a SPECT system has been constructed and tested $[25,26]$ at the acceleratorbased BNCT facility of the University of Birmingham [9]. The device was composed of four independent cylindrical $\mathrm{LaBr}_{3}(\mathrm{Ce})$ scintillators $(2.54 \mathrm{~cm} \times 2.54 \mathrm{~cm})$ coupled to photomultiplier tubes, collimator, shielding and displacement system. The collimator was built in lead with four straight bores $0.5 \mathrm{~cm}$ diameter and $30 \mathrm{~cm}$ length providing a geometrical spatial resolution of 1 $\mathrm{cm}$ at a distance of $30 \mathrm{~cm}$. The shielding has been constructed with successive layers, from outside to inside: a layer of a mixture of paraffin and lithium carbonate to moderate and absorb neutrons; a cadmium layer for further shielding of thermal neutrons; a lead layer for shielding gammas and a final layer of lithium carbonate enriched in ${ }^{6} \mathrm{Li}$ for absorbing the remaining thermal neutrons. The whole system was mounted on motorized rails remotely controlled. A 9 $\mathrm{cm}$ radius cylindrical head phantom filled with water and containing a $3 \mathrm{~cm}$ diameter cylindrical tumor model with $400 \mathrm{ppm}$ of ${ }^{10} \mathrm{~B}$ was used for the experiments.

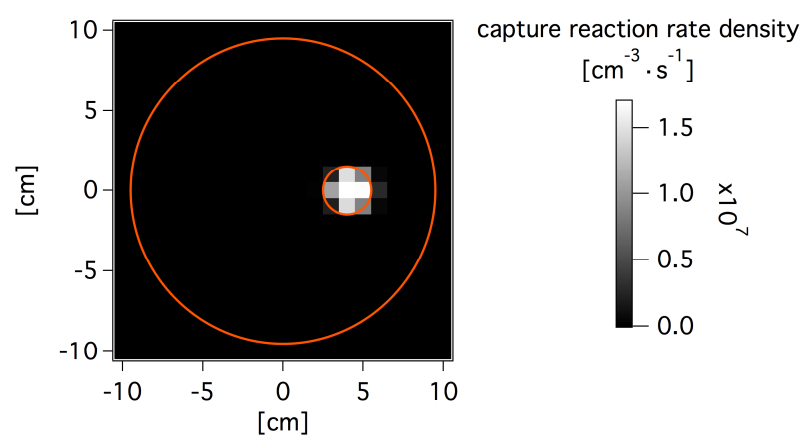

Figure 5: Image of the reconstructed rate of capture reactions in ${ }^{10} \mathrm{~B}$ where red circles indicate the geometrical extension occupied by the tumor and head phantom.

Figure 5 shows the result of the tomographic reconstruction of the experimental data. It is a transversal slice through the cylindrical head and tumor phantoms, $21 \times 21$ pixels of $1 \mathrm{~cm}$ side each, showing the boron density rate distribution. In the tumor, it attains a value of $1.7 \times 10^{7}$ $\mathrm{s}^{-1} \cdot \mathrm{cm}^{-3}$ (error $\sim 15 \%$ ) and presents no significant differences with the MCNP simulation of the tested system, for which the maximum rate was $1.75 \times 10^{7} \mathrm{~s}^{-1} \cdot \mathrm{cm}^{-3}$ (error $\sim 1 \%$ ).

This is the first experimental result of a tomographic acquisition of the boron dose distribution in a phantom irradiated at a facility with a clinical BNCT neutron spectrum. Appropriately scaling the test conditions to more realistic detection efficiency (by increasing the number of detectors), boron concentrations, therapeutic beam intensity and treatment time, this result shows the feasibility of the proposed approach. However it revealed also the need to further reduce the signal background by optimizing the whole system. At present we are working in designing more compact detectors, on the one hand, by reducing the lateral size of the scintillators while increasing it thickness. On the other, we will replace the photomultiplier tubes by pixelated avalanche photodiodes. 


\section{Conclusions}

A $200 \mathrm{kV}$ high-current accelerator prototype is almost ready and an in-air folded $0.7 \mathrm{MV}$ tandem ESQ is being built. Other accelerator configurations like single ended 1.4 MV and 1.4 MV tandem are being pursued. A prototype of a SPECT system for online dosimetry in BNCT based on $\mathrm{LaBr}_{3}(\mathrm{Ce})$ scintillators has been built and tested. We continue working towards the completion of a full accelerator-based BNCT facility.

\section{References}

[1] G.L. Locher, Biological effects and therapeutic possibilities of neutrons, Am J Roentgenol Radium Ther Nucl Med 36 (1936) 1.

[2] D.E. Wazer, R.G. Zamenhof et al., Boron neutron capture therapy, in Mauch P, Loeffler J, editors, Radiation oncology: technology and biology, Saunders, Philadelphia 1994.

[3] H. Tanaka et al. Experimental verification of beam characteristics for cyclotron-based epithermal neutron source, Appl Radiat Isotopes 69 (2011) 1642.

[4] H. Kumada et al., New challenge for advanced BNCT in University of Tsukuba.In the front edge of BNCT development, in proceedings of Sixth Young Researchers BNCT Meeting. 2011, National Tsing Hua University, 132 (2011). ISBN 978-986-03-0321-6.

[5] V. Aleynik et al., BINP accelerator based epithermal neutron source, Appl Rad Isot 69 (2011)1635.

[6] O.E. Kononov et al., Optimization of an accelerator-based epithermal neutron source for neutron capture therapy, Appl Radiat Isotopes 61 (2004) 1009.

[7] S. Halfon et al., High-power liquid-lithium target prototype for AB-BNCT, Appl Radiat Isotopes 69 (2011) 1654.

[8] Ceballos et al., Towards the final BSA modelling for the accelerator-driven BNCT facility at INFN LNL, Appl Radiat Isotopes 69 (2011) 1660.

[9] C.N. Culbertson et al., In-phantom characterisation studies at the Birmingham AcceleratorGenerated epIthermal Neutron Source (BAGINS) BNCT facility, Appl Radiat Isotops 61 (2004) 733.

[10] A.J. Kreiner, M. Baldo, J.R. Bergueiro et al., Accelerator-based BNCT, Appl Radiat Isotopes (2013) http://dx.doi.org/10.1016/j.apradiso.2013.11.064

[11] A.J. Kreiner, J.W. Kwan, A.A. Burlon, et al., A Tandem-Electrostatic-Quadrupole for AcceleratorBased BNCT, Nucl Instrum Meth B 261 (2007) 751.

[12] A.J.Kreiner, V. Thatar Vento, P. Levinas et al., Development of a Tandem-Electrostatic-Quadrupole accelerator facility for BNCT, Appl Radiat Isotopes 67 (2009) S266.

[13] A.J. Kreiner et al., Development of a tandem-electrostatic-quadrupole facility for accelerator-based boron neutron capture therapy, Appl Radiat Isotopes 69 (2011) 1672.

[14] V. Thatar Vento, J. Bergueiro, D. Cartelli, A.A. Valda, A.J. Kreiner, Electrostatic design and beam transport for a folded tandem electrostatic quadrupole accelerator facility for accelerator-based boron neutron capture therapy, Appl Radiat Isotopes 69 (2011) 1649.

[15] D. Cartelli,V. V. Thatar Vento, W. Castell et al., Accelerator tube construction and characterization for a tandem-electrostatic-quadrupole for accelerator-based boron neutron capture therapy, Appl Radiat Isotopes 69 (2011) 1680. 
[16] J. Bergueiro, M. Igarzábal, J.C. Suarez Sandín et al., Development of high intensity ion sources for a Tandem-Electrostatic-Quadrupole facility for Accelerator-Based Boron Neutron Capture Therapy, Appl Radiat Isotopes 69 (2011) 1676.

[17] M.E Capoulat, D.M. Minsky, A.J. Kreiner, Computational assessment of deep-seated tumor treatment capability of the ${ }^{9} \mathrm{Be}(d, n)^{10} \mathrm{~B}$ reaction for accelerator-based Boron Neutron Capture Therapy (AB-BNCT), Physica Medica: European J of Medical Physics 30 (2014) 133.

[18] M.E Capoulat, M.S. Herrera, D.M. Minsky, A.J. Kreiner, The ${ }^{9} B e(d n)^{10} B$ reaction as a neutron source for Boron Neutron Capture Therapy, in this conference proceedings.

[19] M. Suarez Anzorena, L. Gagetti, M. del Grosso, A.J. Kreiner, Characterization of Be deposits on $M o, W$ and $C u$ substrates, to implement in a neutron production target for accelerator-based boron neutron capture therapy, Procedia Materials Science, in press.

[20] L. Gagetti, M. Suarez Anzorena, M. del Grosso, A.J. Kreiner, Simulaciones para un sistema de microcanales de un blanco de producciones de neutrones para la terapia por captura neutrónica en boro, in proceedings of $13^{\text {er }}$ Congreso Internacional en Ciencia y Tecnología de Metalurgia y Materiales 2013, August 20-23 2013, Iguazú, Argentina.

[21] D.M. Minsky, A.J. Kreiner, Near threshold ${ }^{7} \mathrm{Li}(p, n)^{7}$ Be reaction for Boron Neutron Capture Therapy, in this conference proceedings.

[22] A.A. Burlon, S. Girola, A.A. Valda, D.M. Minsky, A.J. Kreiner and G. Sánchez, Design of a beam shaping assembly and preliminary modelling of a treatment room for accelerator-based BNCT, Appl Radiat Isotopes 69 (2011) 1688.

[23] S. Girola, A.Valda, D. Minsky, M.E. Capoulat, A.J. Kreiner, G. Sánchez, Diseño de una sala de tratamiento dedicada a la terapia por captura neutrónica en boro (BNCT) basada en un acelerador de partículas, presented in VI Congreso de la Asociación Latinoamericana de Física Médica, August 24-27 2013, Costa Rica; to be published in Revista de ALFIM.

[24] Valda, D. Minsky, A.J. Kreiner et al., Development of a tomographic system for online dose measurements in BNCT (Boron Neutron Capture Therapy), Braz J Phys 35 (2005) 785.

[25] D.M. Minsky, A.A. Valda, A.J. Kreiner et al., Experimental feasibility studies on a SPECT tomograph for BNCT dosimetry, Appl Radiat Isotopes 67 (2009), s179.

[26] D.M. Minsky, A.A. Valda, A.J. Kreiner, S. Green, C. Wojnecki, Z. Ghani, First tomographic image of neutron capture rate in a BNCT facility, Appl Radiat Isotopes 69 (2011) 1858.

[27] W.F.A.R. Verbakel, W. Sauerwein, et al., Boron concentrations in brain during boron neutron capture therapy: in vivo measurements from the phase I trial EORTC 11961 using a $\gamma$-ray telescope, Int J Radiat Oncol Biol Phys 55 (2003) 743.

[28] T. Kobayashi, Y. Sakurai et al., A noninvasive dose estimation system for clinical BNCT based on $P G$-SPECT-conceptual study and fundamental experiments using HPGe and CdTe semiconductor detectors, Med Phys 27 (2000) 2124.

[29] P. Munck af Rosenschöld, D. Minarik et al., Prompt gamma tomography during BNCT - A feasibility study, J Instrum 1 (2006) 1.

[30] M. Manabe, I, Murata, Study on measuring device arrangement method of Array-type CdTe Detector for BNCT-SPECT, in 7th Young Researchers' Boron Neutron Capture Meeting, Granada, Spain. 22nd to 26th September, 2013, Book of Abstracts, p 47.

[31] L.A. Shepp and Y. Vardi, Maximum likelihood reconstruction for emission tomography, IEEE T Med Imaging, MI-1 (1982) 113. 